\title{
Remote sensing of cloud properties using ground-based measurements of zenith radiance
}

Article

Published Version

Chiu, J. C., Marshak, A., Knyazikhin, Y., Wiscombe, W.J., Barker, H.W., Barnard, J.C. and Luo, Y. (2006) Remote sensing of cloud properties using ground-based measurements of zenith radiance. Journal of Geophysical Research, 111 (D16). D16201. ISSN 0148-0227 doi: https://doi.org/10.1029/2005JD006843 Available at https://centaur.reading.ac.uk/16769/

It is advisable to refer to the publisher's version if you intend to cite from the work. See Guidance on citing.

To link to this article DOI: http://dx.doi.org/10.1029/2005JD006843

Publisher: American Geophysical Union

All outputs in CentAUR are protected by Intellectual Property Rights law, including copyright law. Copyright and IPR is retained by the creators or other copyright holders. Terms and conditions for use of this material are defined in the End User Agreement. 


\section{CentAUR}

Central Archive at the University of Reading

Reading's research outputs online 


\title{
Remote sensing of cloud properties using ground-based measurements of zenith radiance
}

\author{
J. Christine Chiu, ${ }^{1}$ Alexander Marshak, ${ }^{2}$ Yuri Knyazikhin, ${ }^{3}$ Warren J. Wiscombe, ${ }^{2}$ \\ Howard W. Barker, ${ }^{4}$ James C. Barnard, ${ }^{5}$ and Yi Luo ${ }^{6}$ \\ Received 1 November 2005; revised 14 March 2006; accepted 25 April 2006; published 18 August 2006.
}

[1] We have conducted the first extensive field test of two new methods to retrieve optical properties for overhead clouds that range from patchy to overcast. The methods use measurements of zenith radiance at 673 and $870 \mathrm{~nm}$ wavelengths and require the presence of green vegetation in the surrounding area. The test was conducted at the Atmospheric Radiation Measurement Program Oklahoma site during September-November 2004. These methods work because at $673 \mathrm{~nm}$ (red) and $870 \mathrm{~nm}$ (near infrared (NIR)), clouds have nearly identical optical properties, while vegetated surfaces reflect quite differently. The first method, dubbed REDvsNIR, retrieves not only cloud optical depth $\tau$ but also radiative cloud fraction. Because of the 1-s time resolution of our radiance measurements, we are able for the first time to capture changes in cloud optical properties at the natural timescale of cloud evolution. We compared values of $\tau$ retrieved by REDvsNIR to those retrieved from downward shortwave fluxes and from microwave brightness temperatures. The flux method generally underestimates $\tau$ relative to the REDvsNIR method. Even for overcast but inhomogeneous clouds, differences between REDvsNIR and the flux method can be as large as 50\%. In addition, REDvsNIR agreed to better than $15 \%$ with the microwave method for both overcast and broken clouds. The second method, dubbed COUPLED, retrieves $\tau$ by combining zenith radiances with fluxes. While extra information from fluxes was expected to improve retrievals, this is not always the case. In general, however, the COUPLED and REDvsNIR methods retrieve $\tau$ to within $15 \%$ of each other.

Citation: Chiu, J. C., A. Marshak, Y. Knyazikhin, W. J. Wiscombe, H. W. Barker, J. C. Barnard, and Y. Luo (2006), Remote sensing of cloud properties using ground-based measurements of zenith radiance, J. Geophys. Res., 111, D16201, doi:10.1029/2005JD006843.

\section{Introduction}

[2] Cloud optical depth $\tau$ is vital for any cloud-radiation parameterization. To estimate $\tau$ from surface measurements, the atmospheric science community has widely used radiative flux measurements from both broadband and narrowband radiometers [Leontieva and Stamnes, 1994, 1996; Min and Harrison, 1996; Boers, 1997; Barker et al., 1998]. This type of method is, however, limited to overcast conditions and at best, gives "effective" values of $\tau$ instead of "local"

\footnotetext{
${ }^{1}$ Joint Center for Earth Systems Technology, University of Maryland Baltimore County, Baltimore, Maryland, USA.

${ }^{2}$ Climate and Radiation Branch, NASA Goddard Space Flight Center, Greenbelt, Maryland, USA.

${ }^{3}$ Department of Geography, Boston University, Boston, Massachusetts, USA.

${ }^{4}$ Cloud Physics Research Division, Meteorological Service of Canada, Downsview, Ontario, Canada.

${ }^{5}$ Pacific Northwest National Laboratory, Richland, Washington, USA.

${ }^{6}$ Canada Centre for Remote Sensing, Natural Resources Canada, Ottawa, Ontario, Canada.
}

Copyright 2006 by the American Geophysical Union. 0148-0227/06/2005JD006843\$09.00 or overhead values [Ricchiazzi et al., 1995; Dong et al., 1997].

[3] Unlike flux instruments, narrow field-of-view (NFOV) radiometers that measure zenith radiance have the potential to provide local estimates of $\tau$. However, there are two major problems with retrieving $\tau$ from monochromatic zenith radiance. First, in 1D plane-parallel radiative transfer theory there are two potential values of $\tau$ for every zenith radiance. Figure 1 demonstrates that fact, showing that it is impossible, in general, to unambiguously retrieve $\tau$ from a one-channel NFOV radiometer. Second, the histogram of actual observations (solid line in Figure 1) from a one-channel NFOV radiometer reveals that because of three-dimensional (3-D) effects, some radiances may exceed those permitted by 1-D models. This results in $\tau$ being irretrievable for those zenith radiances.

[4] Marshak et al. [2000] and Barker and Marshak [2001] proposed different approaches to reduce the retrieval ambiguity of radiance-based methods. Marshak et al. [2000] estimated $\tau$ from two channels $[673 \mathrm{~nm}$ (red) and $870 \mathrm{~nm}$ (near infrared (NIR))] instead of just one. The underlying principle of their method is that these two channels have similar cloud properties but strong spectral 


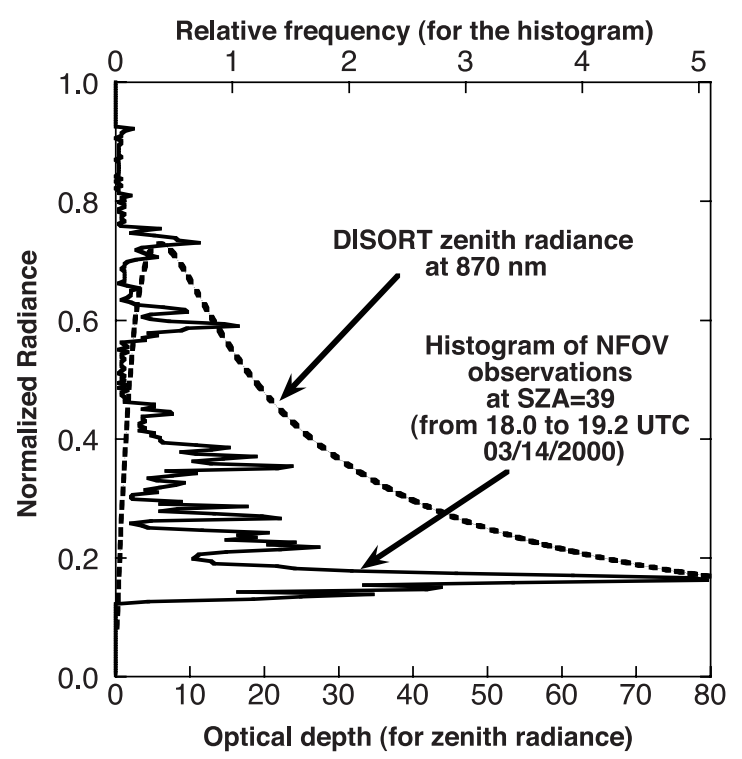

Figure 1. Downward 870-nm radiances versus cloud optical depth (bottom $x$ axis) calculated by the 1-D radiative transfer model DISORT [Stamnes et al., 1988] with a surface albedo of 0.271. Coplotted solid curve is a histogram (top $x$ axis) of one-channel NFOV 870-nm radiances measured at the Atmospheric Radiation Measurement (ARM) Program Oklahoma site from 1800 to 1912 UTC, 14 March 2000.

contrast in vegetated surface reflectance. In an attempt to isolate information pertaining to $\tau$, and by analogy with the normalized difference vegetation index (NDVI) [Tucker, 1979], they introduced a normalized difference cloud index (NDCI) as the ratio of the difference to the sum of normalized zenith radiances at the two channels. However, it was found that $\tau$ could vary considerably while NDCI remained unchanged.

[5] Instead of using a single index like NDCI, Marshak et al. [2004] directly utilized radiance observations on the red versus NIR plane (see Figure 2). Since most vegetated surfaces are dark at red wavelengths and bright at NIR wavelengths, points above the diagonal correspond to cloudy situations due to surface-cloud interactions, while points below the diagonal correspond to clear sky. Since the surface is dark in the red region, having the same red radiances at points $\mathrm{A}$ and $\mathrm{B}$ indicates that they have the same values of $\tau$. However, they have different radiances in the NIR region. Clearly, more surface-cloud interactions occur and more photons reach the ground for point $\mathrm{B}$. This indicates that point $\mathrm{B}$ corresponds to a smaller cloud fraction than point A. This can all be made more quantitative in the method referred to hereinafter as "REDvsNIR", which retrieves both optical depth and "effective" cloud fraction from a point in the red versus NIR plane. Note that points $\mathrm{A}$ and $\mathrm{C}$ in Figure 2 have the same NDCI but correspond to different values of $\tau$ and effective cloud fraction. This is an example of the nonuniqueness problem with the NDCI method, referred to earlier.

[6] The concept of the method proposed by Barker and Marshak [2001], and studied theoretically by Knyazikhin et al. [2005], also relies on this strong spectral difference for surface-cloud interactions. This method coupled zenith radiances with flux measurements to retrieve $\tau$. Thus this method is referred to as "COUPLED". It has been tested for model-simulated clouds and associated radiation fields. To assess the performance of this method in more realistic conditions, Barker et al. [2004] have evaluated $\tau$ retrievals with cloud model-generated data that release the frozen turbulence assumption originally used by Barker and Marshak [2001].

[7] The objective of this paper is to assess the REDvsNIR and COUPLED methods using Atmospheric Radiation Measurement (ARM) Program observations. The ARM program deployed a surface-based two-channel Narrow Field-of-View Radiometer (2NFOV) at the Oklahoma site in September 2004. This radiometer has a $5.7^{\circ}$ field of view and measures downwelling zenith radiance at 673 and $870 \mathrm{~nm}$ with 1-s sampling resolution. With such rapid data, we are able, for the first time, to capture changes in cloud structure.

[8] In an attempt to check whether 2NFOV measurements contain biases, section 2 compares $2 \mathrm{NFOV}$ measurements with CIMEL Sun photometer measurements of the Aerosol Robotic Network (AERONET). Section 3 briefly reviews the methodologies of the REDvsNIR and the COUPLED methods, followed by sensitivity tests in section 4. Section 5 compares our retrievals with those from the ARM MultiFilter Rotating Shadowband Radiometer (MFRSR) and Microwave Radiometer (MWR).

\section{Comparisons With the CIMEL}

[9] Since the accuracy of AERONET's CIMEL measurements is held to a high standard [Holben et al., 1998], the ARM 2NFOV radiances are compared to the CIMEL in order to quantify any biases in the 2NFOV data. The CIMEL Sun photometer is a ground-based radiometer used for aerosol studies that looks directly toward the sun, has a $1.2^{\circ}$ field of view, and four wavelength filters at 440,670 , 870 , and $1020 \mathrm{~nm}$. When clouds block the sun, CIMEL switches to "cloud mode" where it takes 10 measurements

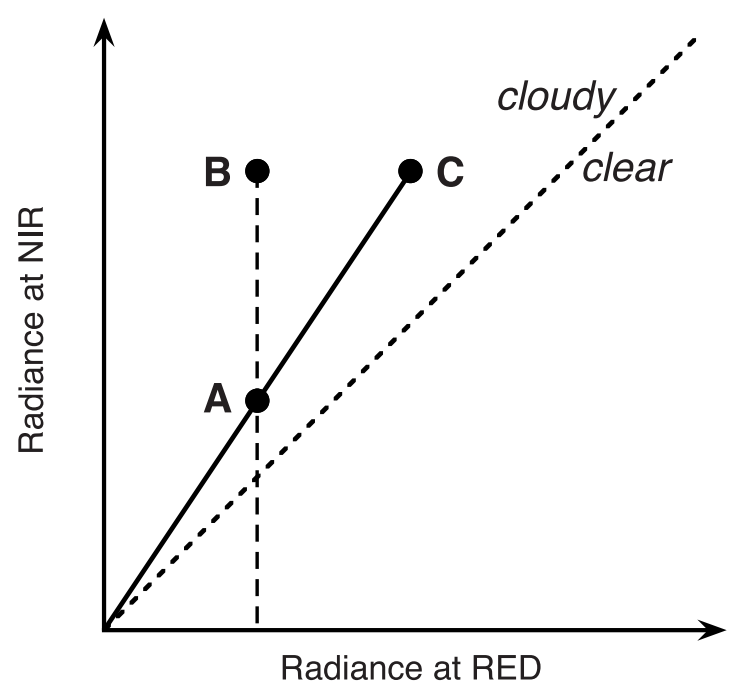

Figure 2. Schematic illustration of the REDvsNIR method for retrieving cloud optical depth and cloud fraction. 


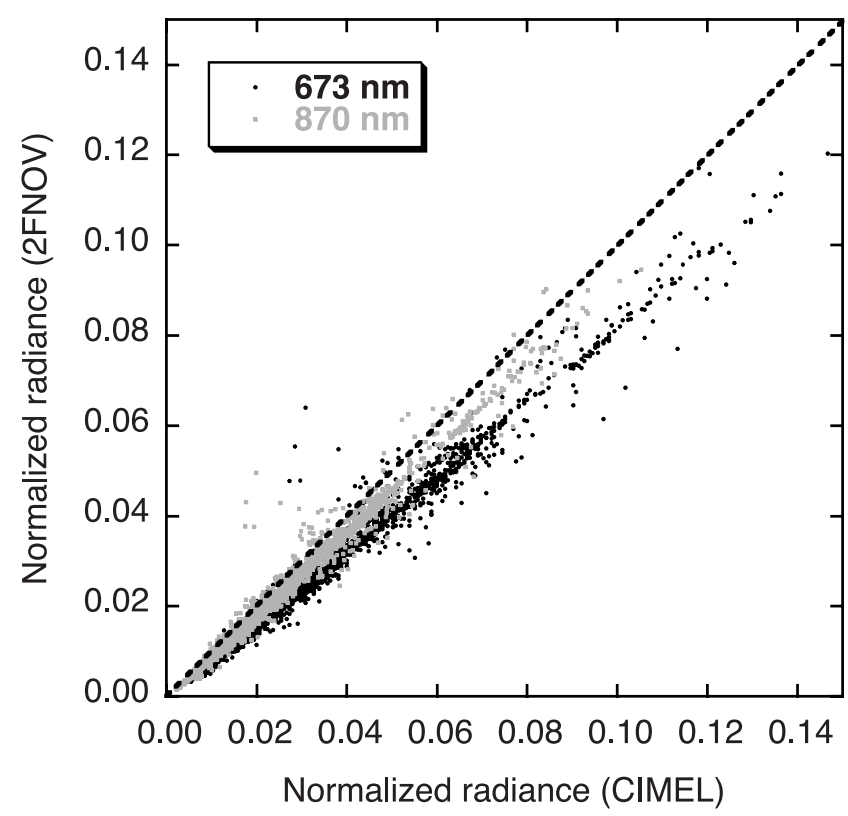

Figure 3. Scatterplot of ARM two-channel NFOV measurements versus CIMEL at channels 673 and $870 \mathrm{~nm}$. Data were collected at the ARM Oklahoma site during 330 November 2004.

of zenith radiance with 9-s temporal resolution [Marshak et al., 2004].

[10] Figure 3 shows 3000 ARM 2NFOV radiances versus CIMEL measurements at the ARM Oklahoma site for the period 3-30 November 2004. This plot shows that these two data sets correlate well, but the ARM 2NFOV radiometer tends to underestimate zenith radiance in the red and NIR regions by about $20 \%$ and $10 \%$, respectively. On the basis of regression analyses, $2 \mathrm{NFOV}$ zenith radiances were adjusted using

$$
\begin{gathered}
I_{\text {red,adj }}=\left(I_{\text {red,obs }}+0.000587\right) / 0.82 \\
I_{\text {NIR,adj }}=\left(I_{\text {NIR,obs }}+0.000400\right) / 0.92,
\end{gathered}
$$

where the subscript $o b s$ represents original measured zenith radiances, and adj is for radiances after the adjustment. This adjustment was necessary in order to make a meaningful comparison between our retrievals and those from other instruments. Retrieval results shown in this paper are based on the adjusted radiances.

\section{Retrieval Methods}

[11] In this section we briefly review both the REDvsNIR and the COUPLED methods. Both methods retrieve overhead cloud optical properties in any cloud situation. The methods use measurements of zenith radiance at 673 and $870 \mathrm{~nm}$ wavelengths and require the presence of green vegetation in the surrounding area. In addition to radiance, the COUPLED method uses downward flux measurements at the same wavelengths.

\subsection{REDvsNIR}

[12] For plane-parallel clouds over a Lambertian surface, any ground-based measurement of radiance $I$ can be expressed as [Box et al., 1988]

$$
I=I_{0}+\frac{\rho I_{s} T_{0}}{1-\rho R} .
$$

The first term on the right hand side, $I_{0}$, is downward radiation calculated over a nonreflecting (black) surface, while the second term is radiation introduced by interactions between clouds and the underlying surface. The cloudsurface interactions are fully determined by $\rho, T_{0}, R$, and $I_{s}$, where $\rho$ is albedo of the underlying surface; $T_{0}$ is transmittance of monochromatic radiance over a black surface; $R$ is spherical albedo of clouds given uniform, isotropic illumination from below; and finally, $I_{s}$ is radiance of a radiation field generated by an isotropic source $1 / \pi$ located at the surface.

[13] Approximating $T_{0}$ with

$$
T_{0}=1-A_{c}+A_{c} \cdot T_{0, p p}
$$

where $A_{c}$ is cloud fraction and $T_{0, p p}$ is total transmittance over a black surface in a plane-parallel assumption, we can rewrite (2) as an explicit function of cloud optical depth $\tau$ and $A_{c}$. For the red and NIR regions, we obtain

$$
\begin{gathered}
I_{\text {red }}\left(\tau, A_{c}\right)=I_{0, \text { red }}(\tau)+\frac{\rho_{\text {red }} I_{s, r e d}(\tau) \cdot\left[1-A_{c}+A_{c} T_{0, p p, r e d}(\tau)\right]}{1-\rho_{\text {red }} R_{\text {red }}(\tau)} \\
I_{N I R}\left(\tau, A_{c}\right)=I_{0, N I R}(\tau)+\frac{\rho_{N I R} I_{s, N I R}(\tau) \cdot\left[1-A_{c}+A_{c} T_{0, p p, N I R}(\tau)\right]}{1-\rho_{N I R} R_{N I R}(\tau)} .
\end{gathered}
$$

Note that it was assumed that the dependency on $A_{c}$ only comes from (3). This $A_{c}$ is not a real cloud fraction, but rather a "radiatively effective" value that forces 3-D measurements to fit into 1-D plane-parallel radiative transfer calculations. Detailed explanations and discussions can be found in the work by Marshak et al. [2004].

[14] As expected from (4), surface albedo has a strong impact on radiances in a forward problem, and thus on retrieved $\tau$ and $A_{c}$ in the inverse problem. We will discuss it separately in section 3.3. To calculate $I_{r e d}$ and $I_{N I R}$ from (4) over a reasonable range of $\tau$ and $A_{c}$, we used DISORT assuming an $8 \mu \mathrm{m}$ effective radius that was typical for cloud droplets at the ARM Oklahoma site. By comparing computed $I_{r e d}$ and $I_{N I R}$ of lookup tables with measurements, we can then retrieve both cloud optical depth and effective cloud fraction simultaneously.

\subsection{COUPLED}

[15] This section outlines a number of key elements in the COUPLED method that combines zenith radiance with flux measurements to retrieve cloud optical depth [Barker and Marshak, 2001; Knyazikhin et al., 2005]. For plane-parallel clouds over a horizontally homogeneous Lambertian sur- 
face with a surface albedo $\rho$, transmittance $T$ can be rewritten as [Petty, 2004, p. 413]

$$
T=\frac{T_{0}}{1-\rho R},
$$

where $T_{0}$ and $R$ are defined in (2). Combining (2) and (5) gives

$$
\begin{gathered}
I_{\text {red }}=I_{0, \text { red }}+\rho_{\text {red }} T_{\text {red }} I_{s, \text { red }} \\
I_{\text {NIR }}=I_{0, \text { NIR }}+\rho_{\text {NIR }} T_{\text {NIR }} I_{s, \text { NIR }} .
\end{gathered}
$$

If clouds have identical optical properties in these two channels then $I_{0, \text { red }}=I_{0, \mathrm{NIR}}$ and $I_{\mathrm{s}, \mathrm{red}}=I_{\mathrm{s}, \mathrm{NIR}}=I_{\mathrm{S}}$. Thus from (6) we can derive

$$
I_{\text {NIR }}-I_{\text {red }}=\left(\rho_{\text {NIR }} T_{\text {NIR }}-\rho_{\text {red }} T_{\text {red }}\right) \cdot I_{s}(\tau) .
$$

[16] For a homogeneous Lambertian surface with a surface albedo $\rho$, upwelling flux $F_{u p}$ measured on the ground relates to transmittance $T$ as

$$
F_{u p}=\rho T F_{0},
$$

where $F_{0}$ is solar irradiance at the TOA at a given wavelength. Let $F^{\uparrow}$ be the upward flux normalized by $F_{0}$. For simplicity, we also normalize $I_{\text {red }}, I_{\mathrm{NIR}}$, and $I_{s}$ leaving the same notations, so that (7) can be rewritten as

$$
I_{N I R}-I_{\text {red }}=\left(F_{N I R}^{\uparrow}-F_{r e d}^{\uparrow}\right) \cdot I_{S}(\tau),
$$

or

$$
I_{S}(\tau)=\frac{I_{N I R}-I_{\text {red }}}{F_{N I R}^{\uparrow}-F_{r e d}^{\uparrow}}
$$

where all quantities are assumed dimensionless hereinafter.

[17] How can (10) be justified for application to inhomogeneous clouds? For horizontally inhomogeneous clouds, (9) can be rewritten as [Knyazikhin et al., 2005]

$$
I_{N I R}(x)-I_{\text {red }}(x)=\int_{x^{\prime} \in S}\left[F_{N I R}^{\uparrow}\left(x^{\prime}\right)-F_{r e d}^{\uparrow}\left(x^{\prime}\right)\right] J\left(x, x^{\prime}\right) d x^{\prime},
$$

where $S$ is the underlying surface; $F_{r e d}^{\uparrow}\left(x^{\prime}\right)$ and $F_{N I R}^{\uparrow}\left(x^{\prime}\right)$ are upward fluxes at location $x^{\prime}$ on the surface; $J\left(x, x^{\prime}\right)$ is the wavelength-independent probability that a photon from an isotropic source $1 / \pi$ located at $x^{\prime}$ arrives at location $x$ after the surface-cloud interaction. For any wavelength $\lambda$, Knyazikhin et al. [2005] defined a bottom-of-atmosphere reflectance at location $x$ as

$$
r_{\lambda}(x)=\frac{\int_{x^{\prime} \in S} F_{\lambda}^{\uparrow}\left(x^{\prime}\right) J\left(x, x^{\prime}\right) d x^{\prime}}{F_{\lambda}^{\uparrow}(x)},
$$

where the numerator describes the surface-cloud interactions when clouds are illuminated from below by horizontally inhomogeneous isotropic sources $F_{\lambda}^{\uparrow}\left(x^{\prime}\right)$. While the upwelling flux $F_{\lambda}^{\uparrow}\left(x^{\prime}\right)$ can vary significantly, the ratio (12) will not necessarily have a large variation. As shown by Knyazikhin et al. [2005, pp. 640-641], $r_{\lambda}(x)$ approximates the maximum eigenvalue of the linear operator defined by the numerator of (12). Since in (12) the kernel $J\left(x, x^{\prime}\right)$ is wavelength-independent, the maximum eigenvalue is also wavelength-independent. Therefore it can be assumed that $r_{\lambda}(x)=r(x)$. Substituting (12) into (11) gives

$$
r(x) \approx \frac{I_{N I R}(x)-I_{r e d}(x)}{F_{N I R}^{\uparrow}(x)-F_{r e d}^{\uparrow}(x)} .
$$

Note that a simple combination of wavelength-dependent radiances and fluxes in (13) eliminates wavelength dependency and relates only to cloud structure above $x$. Comparing with the ratio in (10) for a plane-parallel geometry, we can write

$$
I_{s}(x, \tau) \approx \frac{I_{N I R}(x)-I_{r e d}(x)}{F_{N I R}^{\uparrow}(x)-F_{r e d}^{\uparrow}(x)}
$$

which justifies the use of (10) for horizontally inhomogeneous clouds.

[18] How can $F_{\text {red }}^{\uparrow}(x)$ and $F_{\text {NIR }}^{\uparrow}(x)$ be measured? Since MFRSR provides downwelling fluxes only, (14) cannot be applied directly to retrieve cloud optical depth. Models of the two-point $J\left(x, x^{\prime}\right)$ correlation function are needed to relate downwelling and upwelling fluxes in a 3-D environment. For a plane-parallel geometry with a homogeneous Lambertian surface they are related simply as $F_{\lambda}^{\uparrow}(x)=\rho_{\lambda} F_{\lambda}^{\downarrow}(x)$. Since in the general case such models of $J$ are not yet available, we approximate $F_{\lambda}^{\uparrow}(x)$ using a simple technique proposed by Barker and Marshak [2001]. This technique integrates measured downwelling fluxes over a given time interval using weighting functions that account for cloud base altitude and cloud advection rate. Once $F_{\lambda}^{\uparrow}(x)$ is calculated, $I_{s}$ is derived from (14) and used in lookup tables to retrieve cloud optical depth.

\subsection{Surface Reflectance}

[19] Since REDvsNIR and COUPLED rely on cloudsurface interactions, the spectral contrast in surface reflectance is crucial for the success of these two methods. Note that not only does the difference in spectral surface reflectances matter here; their absolute values do too. By ratioing the reflectance difference between these two channels by their sum, NDVI presents useful information on such a surface reflectance contrast. In general, a larger NDVI value indicates a more significant spectral contrast in surface reflectance. For instances, for $\rho_{\text {red }}=0.0$ and $\rho_{\mathrm{NIR}}=0.5$, NDVI $=(0.5-0.0) /(0.5+0.0)=1$. This index reduces to 0.5 for $\rho_{\text {red }}=0.1$ and $\rho_{\text {NIR }}=0.3$. Figure 4 shows NDVI values for the ARM Oklahoma site during 2004 as estimated from the Moderate Resolution Imaging Spectroradiometer (MODIS). During the observation period, NDVI values were between 0.35 and 0.4 , which was not ideal for our retrieval purpose. Nevertheless, evaluations for this period still help us assess the performance of all methods, and identify problems.

[20] In this paper, we used surface reflectivity parameters provided by the Canada Centre for Remote Sensing, which 


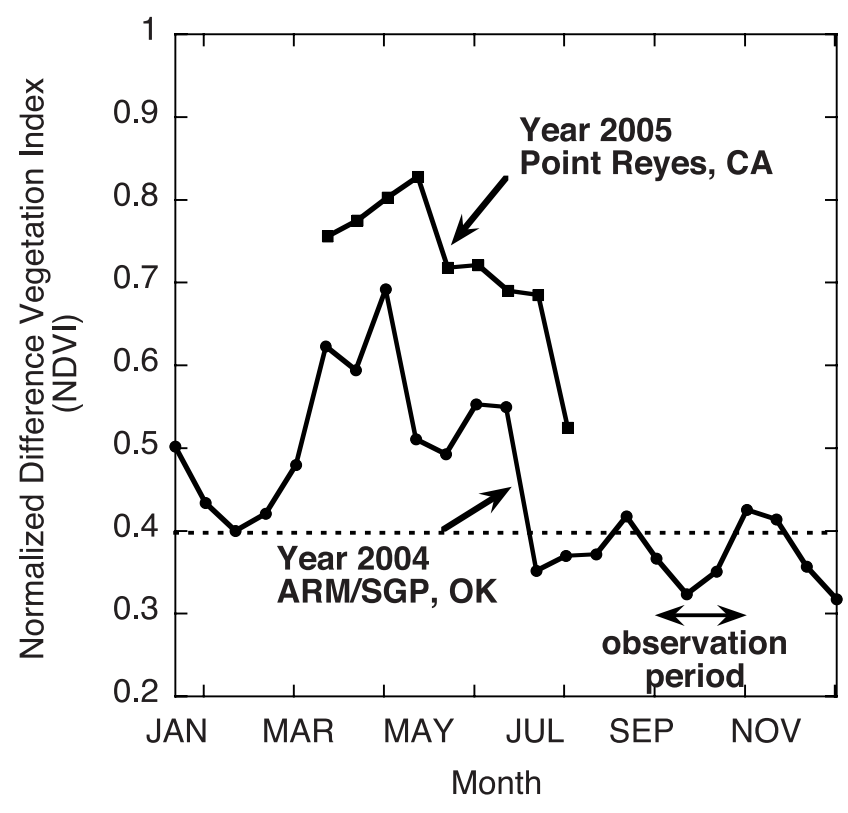

Figure 4. Normalized difference vegetation index (NDVI) from MODIS for the ARM Oklahoma site, 2004, and for Point Reyes, California, 2005. The dashed line represents the threshold of 0.4 . A NDVI smaller than this threshold often indicates an unsuitable surface reflectance contrast between red and NIR channels.

were optimally derived from a number of satellites [Luo et al., 2005]. These parameters were provided every 10 days at a $500 \mathrm{~m}$ resolution. Uncertainties in surface albedo are assumed to be $10 \%$ in the red and 5\% in the NIR. These are close to the retrieval accuracies of current surface reflectance products that are available from satellite measurements [Schaaf et al., 2002]. How these uncertainties impact retrieved $\tau$ is discussed next.

\section{Sensitivity Tests}

[21] This section studies how uncertainty in measurements impacts our retrievals for both the REDvsNIR and COUPLED methods. Note that Beaulne et al. [2005] studied the sensitivity of the COUPLED method to instrument noise using simulated data. Here we scrutinize the assumption made in the COUPLED method that downward red and NIR radiances are identical for a black surface. We also test how sensitive the COUPLED method is to uncertainties in radiance, flux, and surface albedo, using equation (14) rather than model simulations. Results show that the COUPLED method under some conditions becomes unstable, which is a common drawback for a method that retrieves $\tau$ from a ratio like equation (14).

\subsection{REDvsNIR}

[22] For REDvsNIR, uncertainty comes from measurements of zenith radiance and surface reflectance. Assuming $1 \%$ uncertainty in zenith radiance in both red and NIR regions, we found less than $4 \%$ uncertainty in retrieved $\tau$ when clouds were overcast with $\rho_{\text {red }}=0.1$ and $\rho_{\mathrm{NIR}}=0.3$. This retrieval uncertainty becomes smaller with increasing spectral contrast in surface reflectance.
[23] Marshak et al. [2004] demonstrated how retrieved $\tau$ responded to errors in surface albedo using uncertainty of $25 \%$ in the red region and $8 \%$ in the NIR for values of $\rho_{\text {red }}=$ 0.1 and $\rho_{\mathrm{NIR}}=0.3$. They found an average error of $2-3 \%$ in retrieved $\tau$ when uncertainties in these two spectral regions have the same sign. This retrieval error increased to $5-10 \%$ when uncertainties have different signs. When $\rho_{\text {red }}=0.1 \pm$ $10 \%$ and $\rho_{\mathrm{NIR}}=0.3 \pm 5 \%$ are used, retrievals show an average $2 \%$ retrieval error. However, assuming the same $5-10 \%$ uncertainty in $\rho_{\text {red }}$ and $\rho_{\text {NIR }}$, this retrieval error increases dramatically as the contrast in surface reflectances diminishes.

\subsection{COUPLED}

[24] For the COUPLED method, in addition to uncertainty in radiance measurements and surface albedo, there are two more sources of error: (1) the assumption of $I_{0, \text { red }}=I_{0, \mathrm{NIR}}$, and (2) the estimation of upward fluxes at the cloud base from a time series of downward flux measurements at the surface. The latter error source is included here in the uncertainty of the upward fluxes $F_{\lambda}^{\uparrow}$. The former source will be discussed next in more details.

\subsubsection{Effects of Assumption $\boldsymbol{I}_{\mathbf{0}, \mathrm{red}}=\boldsymbol{I}_{\mathbf{0}, \mathrm{NIR}}$}

[25] As mentioned in section 3.2, the COUPLED method is based on an assumption that downward radiances in the red and NIR regions are equal for a black surface (i.e., $I_{0}$ ). To quantify the retrieval error due to this assumption, two sets of synthetic data were tested. The first data set was generated using the Henyey-Greenstein phase function for cloud droplets with asymmetry factors of 0.856 and 0.851 at the red and NIR wavelengths, respectively. The second data set was based on Mie phase functions assuming an effective radius of $8 \mu \mathrm{m}$. These two data sets have the same asymmetry factors.

[26] In the general case of $I_{0, \text { red }} \neq I_{0, \mathrm{NIR}}$, it follows from (6) and the normalized counterpart of (8) that

$$
I_{s}=\frac{I_{N I R}-I_{\text {red }}}{F_{N I R}^{\uparrow}-F_{r e d}^{\uparrow}}+\frac{I_{0, \text { red }}-I_{0, N I R}}{F_{N I R}^{\uparrow}-F_{r e d}^{\uparrow}},
$$

where the second term on the right hand side accounts for $I_{0, \text { red }} \neq I_{0, \text { NIR }}$. The COUPLED method works only if this term is negligible compared to the first one on the right hand side. In that case no substantial retrieval errors are introduced by assuming $I_{0, \text { red }}=I_{0, \mathrm{NIR}}$.

[27] The percentage of the second term relative to $I_{s}$ is plotted in Figure 5. On the basis of the data generated from the Henyey-Greenstein phase function, for a significant surface reflectance contrast (Figure 5a), the assumption of $I_{0, \text { red }}=I_{0, \mathrm{NIR}}$ introduces errors that are $<10 \%$. This error goes up significantly with a decreasing contrast in surface albedo (Figure 5b), though the error is reduced with a decreasing cloud fraction (not shown). When the Mie phase functions were used, the error increased (Figures $5 \mathrm{c}$ and $5 \mathrm{~d}$ ). For a small contrast in surface albedo (e.g., $\rho_{\text {red }}=0.1, \rho_{\mathrm{NIR}}=$ 0.3 ), the assumption $I_{0, \text { red }}=I_{0, \mathrm{NIR}}$ contributes more than $25 \%$ error in $I_{S}$ for overcast, thick clouds (Figure $5 \mathrm{~d}$ ). This error becomes even greater when the solar zenith angle (SZA) is large. Note that since $I_{0, \text { red }}>I_{0, \text { NIR }}$ for all $\tau$ except very small ones, the second term in (15) is positive. Thus the assumption of $I_{0, \text { red }}=I_{0, \mathrm{NIR}}$ introduces a bias that always underestimates $\tau$. 

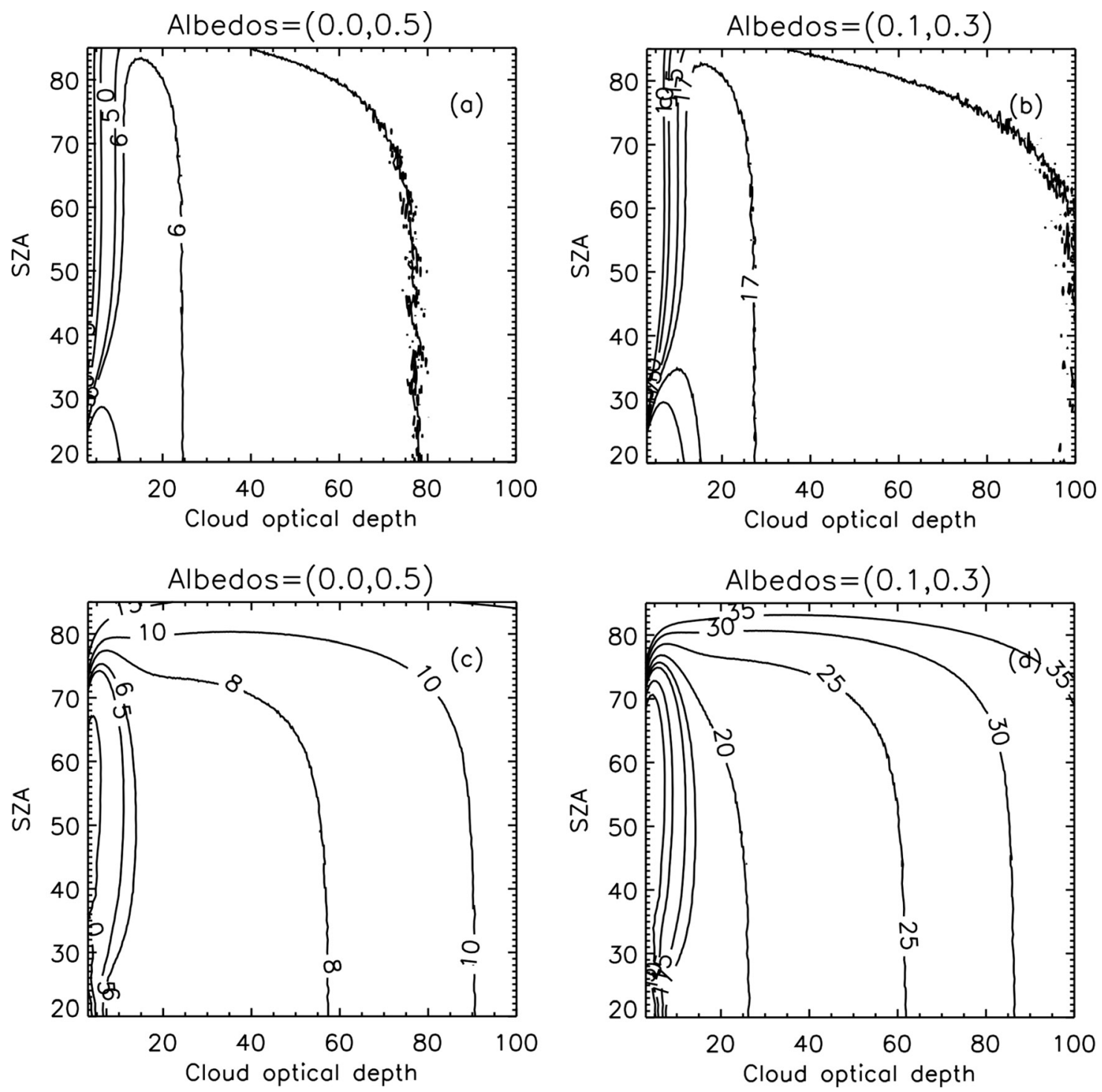

Figure 5. Contours of error percentages due to the assumption of $I_{\mathrm{o}, 1} \sim I_{\mathrm{o}, 2}$ in the COUPLED method when $A_{c}=1$. Plots are based on (top) the Henyey-Greenstein phase function for (a) $\left(\rho_{1}, \rho_{2}\right)=(0.0,0.5)$ and (b) $\left(\rho_{1}, \rho_{2}\right)=(0.1,0.3)$ and (bottom) Mie phase functions for $(\mathrm{c})\left(\rho_{1}, \rho_{2}\right)=(0.0,0.5)$ and $(\mathrm{d})\left(\rho_{1}, \rho_{2}\right)=$ $(0.1,0.3)$.

[28] A possible solution to eliminate this bias is to retrieve $\tau$ directly from lookup tables using the ratio $\left(I_{N I R}-I_{\text {red }}\right) /\left(F_{N I R}^{\uparrow}-F_{\text {red }}^{\uparrow}\right)$ instead of $I_{s}$. However, this ratio is not a monotonic function of $\tau$. The lack of one-to-one relationship begins at different values of $\tau$ depending on surface albedo contrast, cloud fraction, and SZA. In general, this function looses its uniqueness at smaller optical depths when the surface albedo contrast is small, the sky is overcast, and SZA is large.

\subsubsection{Effects of Measurement Uncertainties}

[29] This section aims to evaluate the sensitivity of the COUPLED method to the uncertainty in (1) measurements of both zenith radiance and upward flux and (2) surface albedo. To isolate these two uncertainty sources for COUPLED, we have to assure that $I_{0, \text { red }}=I_{0, \text { NIR }}$ to avoid errors introduced by that assumption (as the previous section described). Therefore we used synthetic measurements that were generated from the Henyey-Greenstein phase function with an asymmetry factor of 0.856 for both channels.

[30] Figure 6a shows the ranges of retrieved cloud optical depths that respond to $1 \%$ uncertainty in both radiances and fluxes with a SZA of $60^{\circ}$. When the sky is overcast $\left(A_{c}=1\right)$ and the surface albedo contrast is significant (e.g., $\rho_{\text {red }}=0.0$, $\left.\rho_{\mathrm{NIR}}=0.5\right), 1 \%$ measurement uncertainty produces roughly a $20-50 \%$ variation in retrievals. Variations of retrievals are dramatically magnified when the surface albedo contrast becomes smaller (e.g., $\left.\rho_{\text {red }}=0.1, \rho_{\text {NIR }}=0.3\right)$. However, for broken clouds (e.g., $A_{c}=0.5$ ), fluctuations in retrieved $\tau$ are reduced since more radiation reaches the surface, thus enhancing surface-cloud interactions.

[31] It is important to point out that the uncertainty in measurements leads to much larger overestimates than underestimates. Consider, for example, the case of $\rho_{\text {red }}=$ $0.1, \rho_{\mathrm{NIR}}=0.3$, and $A_{c}=1$. At the true optical depth of 30 in Figure $6 a, 1 \%$ measurement uncertainty may result in a $30 \%$ 

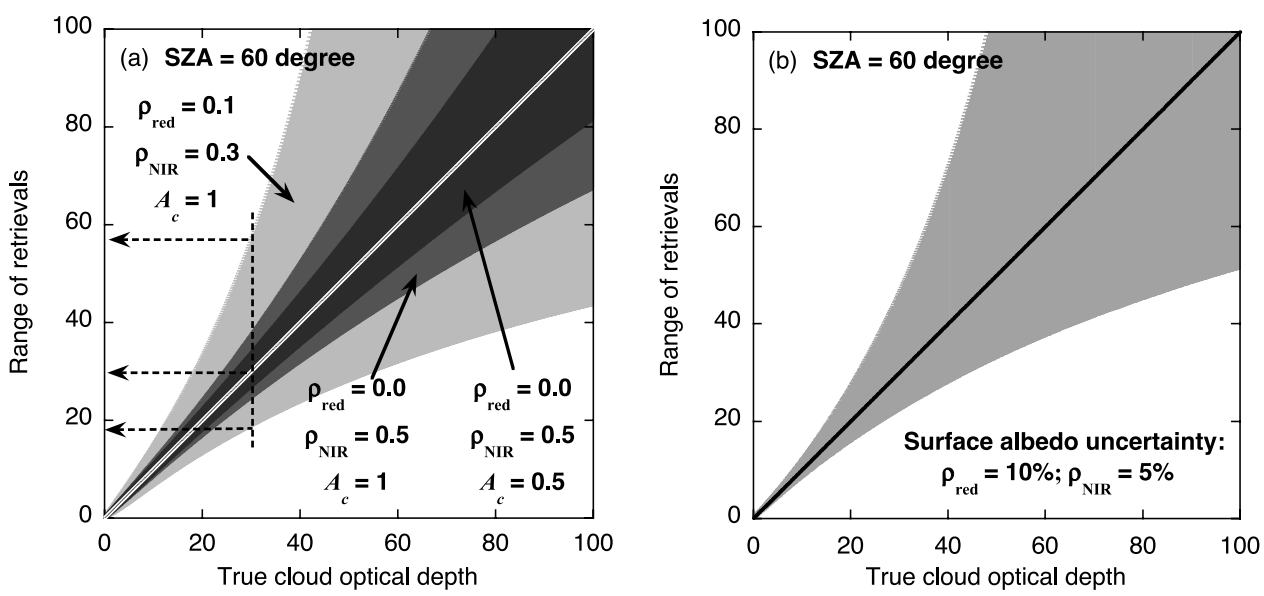

Figure 6. (a) Fluctuations of cloud optical depth retrievals versus true value using synthetic data with $1 \%$ uncertainty in both radiances and fluxes. Note that the histogram of retrievals is skewed to larger cloud optical depths, that is, a positive skewness (not shown). Data were generated using the HenyeyGreenstein phase function with an asymmetry factor of 0.856 for both channels. Shading from outside to inside represents the ranges of retrieved cloud optical depths, based on $\rho_{1}=0.1, \rho_{2}=0.3$, and $A_{c}=1$; $\rho_{1}=0.0, \rho_{2}=0.5$, and $A_{c}=1$; and $\rho_{1}=0.0, \rho_{2}=0.5$, and $A_{c}=0.5$, respectively. (b) Same as Figure 6 a but for $\rho_{1}=0.1 \pm 10 \%, \rho_{2}=0.3 \pm 5 \%$, and $A_{c}=1$.

(retrieved $\tau \approx 20$ ) underestimation or $90 \%$ (retrieved $\tau \approx 56$ ) overestimation. In this example, we assume only $1 \%$ measurement uncertainty. However, according to the ARM instrument handbook, flux measurement uncertainties can be as large as $5 \%$. The achievement of $1 \%$ accuracy in radiance is also quite challenging. Therefore, for the COUPLED method, measurement uncertainties can significantly overestimate cloud optical depths when $\tau>30$ [see also Beaulne et al., 2005].

[32] The sensitivity of the COUPLED method to uncertainty in surface albedo is illustrated in Figure 6b, in which $\rho_{\text {red }}=0.1 \pm 10 \%$ and $\rho_{\text {NIR }}=0.3 \pm 5 \%$. As expected, retrieval uncertainty for thicker clouds is substantially larger than for thinner ones. In short, the COUPLED method is expected to work better for (1) optically thinner, (2) broken clouds above surfaces with (3) a large contrast between red and NIR reflectance. If even one of these three conditions is violated, resulting retrievals can be less reliable. This suggests that retrieval of $\tau$ is an ill-posed problem.

\subsubsection{Joint Effects}

[33] We have demonstrated that the assumption of $I_{\mathrm{o}, \text { red }}=$ $I_{\mathrm{O}, \mathrm{NIR}}$ in the COUPLED method introduces a bias that always underestimates $\tau$; and measurement uncertainties significantly overestimate $\tau$. These biases might be reduced or even cancelled out to yield reasonable retrievals in some circumstances. However, in many cases in which the bias is not eliminated completely, highly unstable overestimations will appear in retrievals, especially for overcast sky situations.

[34] To conclude for the COUPLED method:

[35] 1. The more broken the cloud field is, the more stable the retrieval of cloud optical depth. The method works best for $A_{c} \ll 1$.

[36] 2. The less optically thick clouds are, the more reliable the retrieved optical depths. The method works best for $\tau \leq 30$.
[37] 3. The larger the spectral contrast in surface reflectance, the more reliable and stable the retrievals. The method works best for NDVI $>0.4$.

\section{Results and Comparison With Other Methods}

[38] Other ground-based methods also retrieve optical depth. Two of the most popular are the MFRSR (MultiFilter Rotating Shadowband Radiometer) shortwave flux method and the microwave method [Liljegren and Lesht, 1996; Min and Harrison, 1996; Liljegren et al., 2001; Min et al., 2004]. Here we will compare our REDvsNIR and COUPLED methods with those two methods.

[39] The ARM MFRSR provides 20-s averages of both direct and diffuse solar flux in narrow bands centered at 415 , $500,615,673,870$, and $940 \mathrm{~nm}$. We used MFRSR direct and diffuse transmittance at $415 \mathrm{~nm}$, together with 1-D radiative transfer theory, to retrieve cloud optical depth, similar to the method of Min and Harrison [1996]. 415 nm was chosen because surface reflectance is least at this wavelength and thus minimizes cloud to ground interactions. We cannot expect retrievals from the MFRSR to agree with those from our REDvsNIR method, because the former retrieves a quantity averaged over the sky hemisphere while the latter method retrieves a quantity directly overhead.

[40] We also compared our retrievals with those from microwave-retrieved liquid water path (LWP). The ARM microwave radiometer (MWR) provides 1-s average brightness temperatures at 23.8 and $31.4 \mathrm{GHz}$ and LWP retrievals every $20 \mathrm{~s}$. Its field of view is $5.9^{\circ}$ while the $2 \mathrm{NFOV}$ radiometer's is $5.7^{\circ}$. Since an $8 \mu \mathrm{m}$ effective radius of cloud droplets was used in REDvsNIR and COUPLED, we used the same effective radius to convert microwave LWP to cloud optical depth.

\subsection{Overcast Case}

[41] We chose two overcast periods for study. Table 1 summarizes averages and standard deviations over these periods for the four methods. 
Table 1. Averages and Standard Deviations of Retrieved Cloud Optical Depths Over Two Overcast Time Periods ${ }^{\mathrm{a}}$

\begin{tabular}{lccccc}
\hline & \multicolumn{2}{c}{ 11 November 2004 } & & \multicolumn{2}{c}{ 28 October 2004 } \\
\cline { 2 - 3 } \cline { 5 - 6 } Method & Mean & $\begin{array}{c}\text { Standard } \\
\text { Deviation }\end{array}$ & & Mean & $\begin{array}{c}\text { Standard } \\
\text { Deviation }\end{array}$ \\
\hline REDvsNIR & 15 & 4.6 & & 23 & 12.2 \\
COUPLED & 32 & 16.3 & & 26 & 14.5 \\
MWR & 13 & 4.3 & & 22 & 4.5 \\
MFRSR & 10 & 1.3 & & 16 & 2.2 \\
CIMEL & 16 & 3.8 & & - & - \\
\hline
\end{tabular}

${ }^{\mathrm{a}}$ The first time period is from 1430 to 1500 UTC on 11 November 2004 and the second time period is from 1709 to 1718 UTC on 28 October 2004.

[42] Figure 7a shows time series of retrievals for a visually overcast case, as illustrated by three sky images from the ARM Total Sky Imager (TSI) at times indicated by vertical dashed lines in the plots. The time series of retrieved cloud fractions from REDvsNIR is shown at the top of Figure $7 \mathrm{a}$; values range from 0.4 to 0.9 with a mean of 0.6 and show that the clouds are not homogeneous, which is also evident in the TSI images. Note that there are a few negative cloud fractions due to strong 3-D effects [see Marshak et al., 2004].

[43] Because of the MFRSR's hemispherical field of view, the MFRSR cloud optical depths in Figure 7a fluctuate much less than those from REDvsNIR. Also, Table 1 shows that the average cloud optical depth for REDvsNIR is 15 while that for MFRSR is 10. Because clouds in this case are inhomogeneous (as shown in TSI images and retrieved effective cloud fractions), the MFRSR-method planeparallel assumption is violated and leads to a significantly smaller average cloud optical depth. We find this MFRSR method underestimation to be generally true.

[44] Retrievals from the MWR method show similar fluctuation to those from the REDvsNIR. However, a few retrievals seem to be out of phase with each other, which might be attributed to the uncertainty in effective radius of cloud droplets in the MWR method. Despite this, Table 1
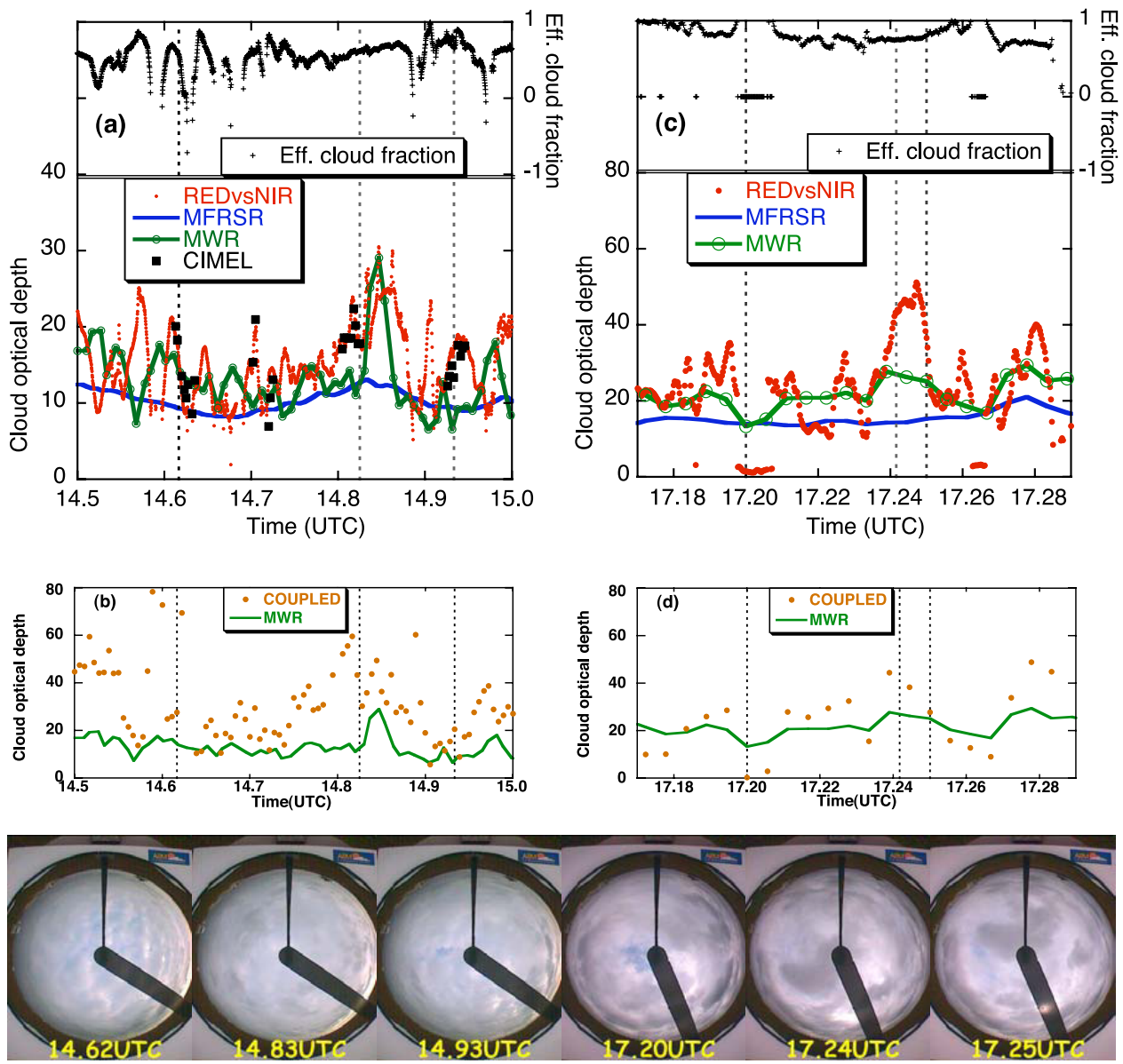

Figure 7. (a) Retrieved cloud optical depths (left $y$ axis) and effective cloud fractions (right $y$ axis) at the ARM Oklahoma site for 11 November 2004. Red dots are retrievals from the REDvsNIR method using the 2NFOV radiance. Blue lines are based on the MFRSR flux method, similar to Min and Harrison [1996]. Green lines are based on the MWR method assuming an 8- $\mu \mathrm{m}$ droplet effective radius. Black squares are CIMEL retrievals from the REDvsNIR method. Sky images are shown below at times indicated by vertical dashed lines in the plots. Solar zenith angles are between $70^{\circ}$ and $75^{\circ}$; surface albedo values are $\rho_{\text {red }}=0.17$ and $\rho_{\text {NIR }}=0.36$. (b) Same as Figure 7a but for retrievals from the COUPLED method (orange dots). (c) and (d) Same as Figures 7a and 7b, respectively, but for 28 October 2004 with $\mathrm{SZA}=52^{\circ}, \rho_{\text {red }}=0.13$, and $\rho_{\mathrm{NIR}}=0.28$. CIMEL observations were not available for this day. 

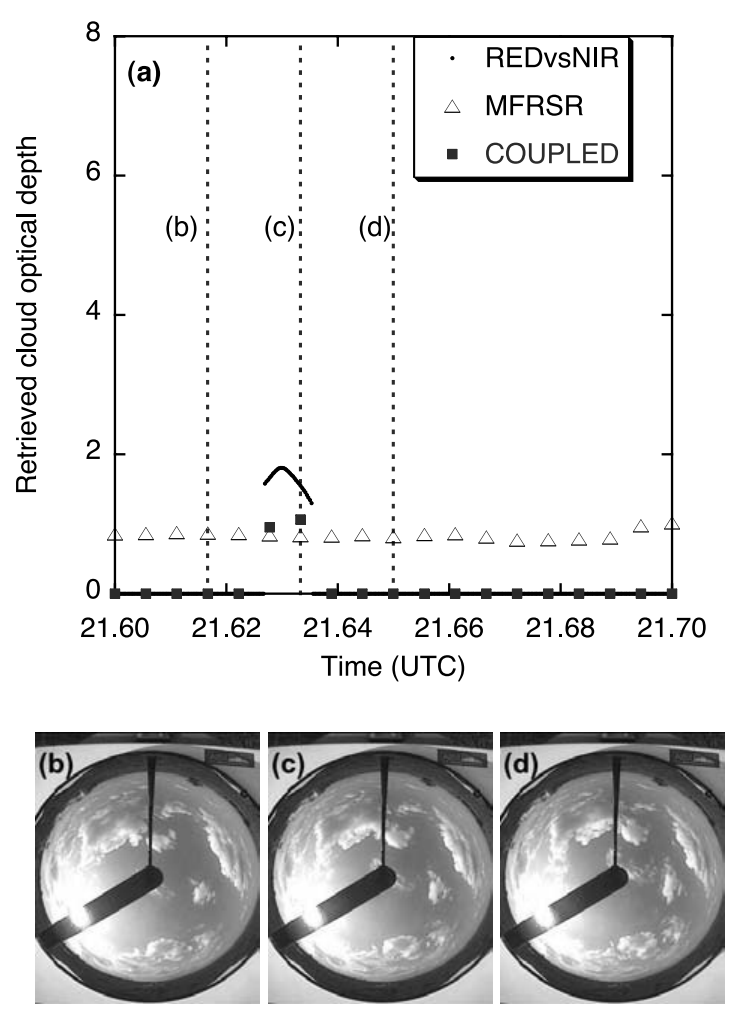

Figure 8. (a) Same as Figure 7 but for 2136:00-2142:00 UTC, 29 September 2004. TSI images were taken at (b) 2137:00, (c) 2138:00, and (d) 2139:00 UTC. Here $\rho_{\text {red }}=$ $0.13, \rho_{\mathrm{NIR}}=0.28$, and $\mathrm{SZA}=61^{\circ}$. MWR measurements were not available for this time period. Note that effective cloud fractions cannot be accurately retrieved for this case (see text for explanations).

shows that the average cloud optical depths from these two methods are close: 15 for REDvsNIR, and 13 for the MWR method.

[45] Figure 7a also plots cloud optical depths retrieved from CIMEL radiance using the REDvsNIR method (as shown by black squares). Not surprisingly, retrievals from the CIMEL and 2NFOV radiance agree very well with each other, since our $2 \mathrm{NFOV}$ radiance was adjusted according to CIMEL measurements.

[46] In section 4.2 we learned that the COUPLED method could become unstable when clouds are thick, overcast, and/or if the spectral contrast in surface albedo is small. This has happened for the overcast sky as shown in Figure $7 \mathrm{~b}$. For this case, clouds are thick and overcast; solar illumination is very oblique with SZA between $70^{\circ}-75^{\circ}$; the contrast in surface reflectance is too low (NDVI $=0.35$; see Figure 4). As a result, the COUPLED method not only becomes unstable but also substantially overestimates $\tau$.

[47] The performance of the COUPLED method is slightly improved with higher solar elevations. Figure $7 \mathrm{c}$ shows $7 \mathrm{~min}$ of observations with $\mathrm{SZA}=52^{\circ}$. Retrieved effective cloud fractions range from 0.6 to 1.0 with a mean of 0.7 , and show quite a few clear-sky scenes. With higher cloud optical depths, this makes the scene less homogeneous than the previous overcast case (panel 7a).
[48] As in the previous case (Figure 7a), Figure 7c shows that retrievals from the MFRSR method have the least fluctuation; and retrievals from the REDvsNIR and MWR methods agree well with each other almost everywhere (see also Table 1). Unlike the previous overcast time period (Figure 7b), Figure 7d shows that the COUPLED method is, in general, more stable here. Retrievals from the COUPLED method now show a similar fluctuation to those from the REDvsNIR and MWR methods.

\subsection{Broken Clouds}

[49] Figure 8 demonstrates retrievals for a thin patchy cloud. This patchy cloud passed by during 2136-2142 UTC and was retrieved by REDvsNIR with an optical depth of 2 . Obviously, the MFRSR method failed to retrieve this patchy cloud, but presented an effective cloud optical depth of surrounding clouds.

[50] It would be interesting to learn about cloud fractions for this case. Unfortunately, we cannot retrieve them accurately here. It is because for small $\tau$, various effective cloud fractions correspond to very similar radiances at both red and NIR wavelengths. Therefore we cannot solve for those cloud fractions and have to leave them as undefined.

[51] Another broken cloud case with rapid cloud advection and evolution is illustrated in Figure 9. Because of similar FOV between the 2NFOV and MWR, we expected great similarity for retrievals from the REDvsNIR and MWR methods. When relatively thick clouds are in the FOV, cloud optical depths retrieved from these two methods
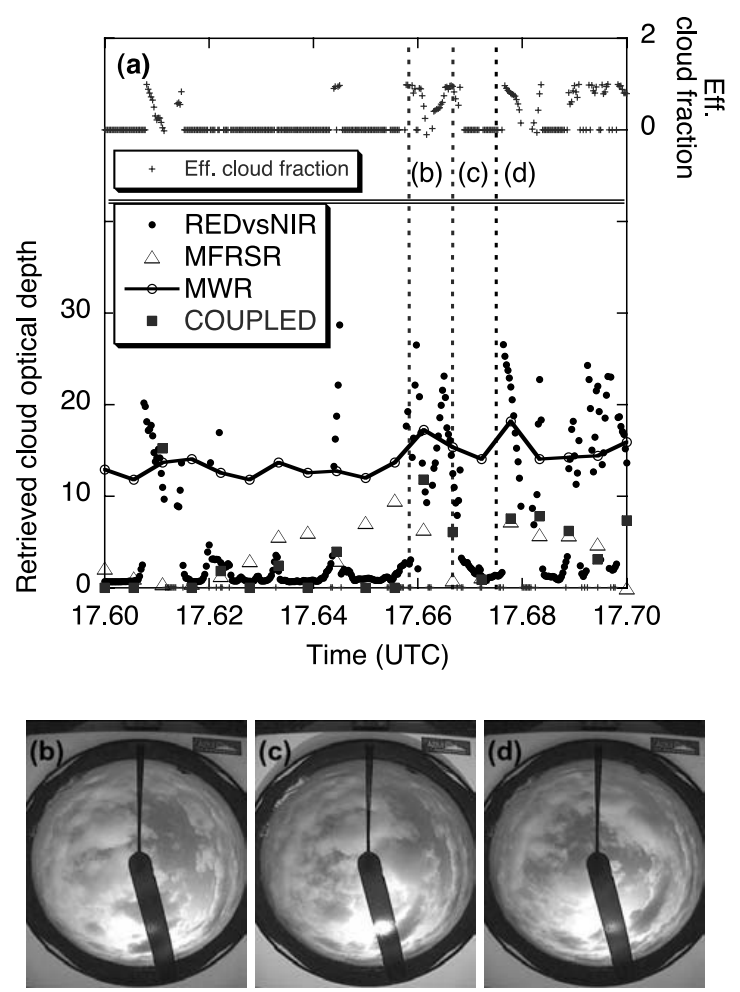

Figure 9. (a) Same as Figure 7 but for 1736:00-1742:00 UTC, 28 October 2004. TSI images were taken at (b) 1739:30, (c) 1740:01, and (d) 1740:30 UTC. Here $\rho_{\text {red }}=0.13, \rho_{\mathrm{NIR}}=0.28$, and $\mathrm{SZA}=51^{\circ}$. 

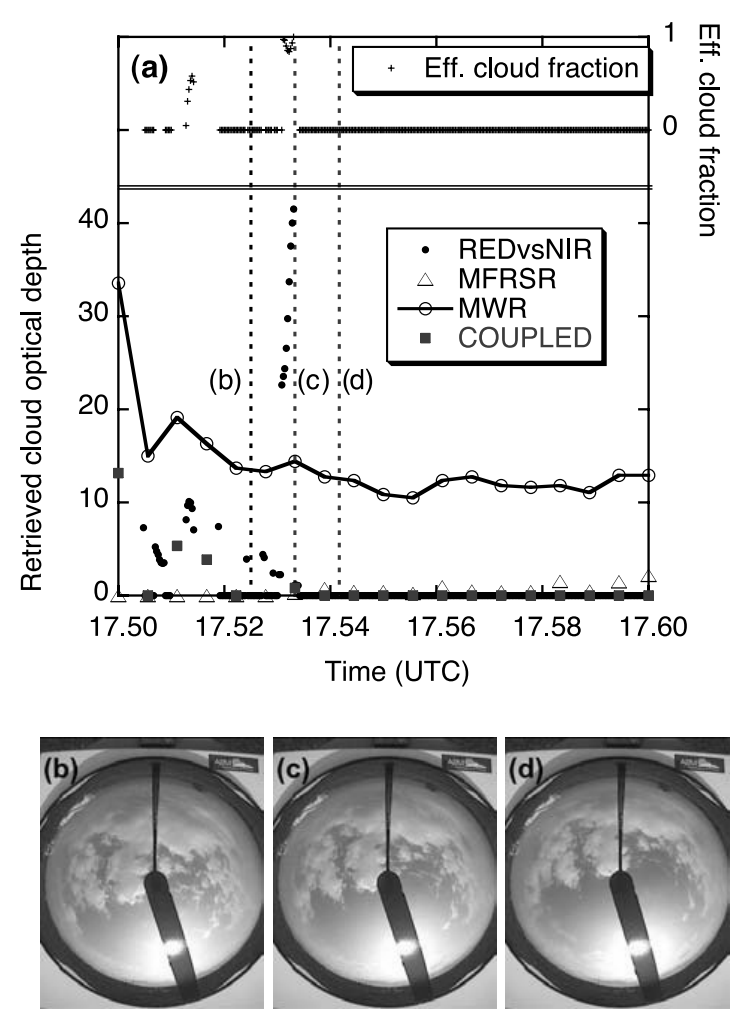

Figure 10. (a) Same as Figure 7 but for 1730:001742:00 UTC, 28 October 2004. TSI images were taken at (b) 1731:30, (c) 1732:00, and (d) 1732:30 UTC. Here $\rho_{\text {red }}=0.13, \rho_{\mathrm{NIR}}=0.28$, and $\mathrm{SZA}=51^{\circ}$.

are very close (as shown in Figures $9 \mathrm{~b}$ and 9c). However, in some cases with thin clouds in the FOV (e.g., Figure 9d), REDvsNIR yields cloud optical depths that are smaller than those for the MWR method.

[52] To quantify differences between the REDvsNIR and MWR methods, we selected coincident measurements of $2 \mathrm{NFOV}$ and MWR. For points with retrieved $A_{c}>0$, we found that the difference in average cloud optical depth between REDvsNIR and the MWR method is only 7\%. For points with $A_{c}=0$ or undefined $A_{c}$ (thin clouds), the average cloud optical depth is 1 for REDvsNIR, while the MWR method yields an average of 13 . We believe that for clearsky and thin cloud situations, this substantial retrieval difference between these methods is attributed to the retrieval uncertainty in the MWR method.

[53] From coincident retrievals of the COUPLED and MFRSR methods, the average cloud optical depth is 4 for both methods, but the COUPLED method retrievals have a larger standard deviation (4.5 compared to 2.9 from MFRSR). On the other hand, the average cloud optical depth for REDvsNIR is 8 .

[54] Note that a number of retrievals from REDvsNIR suddenly jumped from small values (about 3) to very large values (above 15). We found these situations happened when cumulus clouds just passed by and the instrument's field of view was not fully filled by clouds. These problematic situations will be discussed in the next section.

\subsection{Clear-Sky Contamination}

[55] Figure 10 illustrates a potential problem with the "narrow" field of view of the $2 \mathrm{NFOV}$ radiometer, which is not narrow enough in cloud property retrievals. For instance, there are a few unphysical retrieved cloud optical depths (up to 40) around 1732 UTC. Looking at the center of the coincident TSI image, the top left quadrant had some small cumulus clouds at this time, but the other three quadrants were clear. This cloud moved out $30 \mathrm{~s}$ later as shown in the next TSI snapshot. It is evident that there is no cloud thick enough to produce such large optical depths.

[56] This retrieval error is attributed to the clear sky parts of the field of view. The situation with partially cloudy and partially clear within the FOV leads to small radiances at both channels (as illustrated in Figure 11a). REDvsNIR fails since it cannot differentiate this situation from optically thick clouds that also produce small radiances (Figure 11b). This problem, referred to here as the "clear-sky contamination problem", occurs on many other days. Therefore the FOV of the ARM $2 \mathrm{NFOV}$ radiometer has recently been reduced to $1.2^{\circ}$ to lower the probabilities of clear-sky contaminations, and will be further tested in the ARM field campaign.

\subsection{Cloud Edge}

[57] Finally, Figure 12 shows that REDvsNIR fails to retrieve cloud optical depths in some broken cloud scenes because of another problem called "cloud edge problem". This problem occurs when the sun directly illuminates cloud edges (as TSI images show). This strong illumination causes a substantial amount of photons to scatter into the field of view, and results in large radiances that are outside of the area covered by our 2-D lookup table. Although this problem has not occurred often (less than $10 \%$ of data), we expect to review this problem with new $1.2^{\circ}$ field-ofview measurements.

[58] This broken cloud case demonstrates an attractive advantage of the COUPLED method. As Figure 12 shows, a retrieval method that uses only radiances (REDvsNIR) or only fluxes (MFRSR) fails to retrieve cloud optical depth of

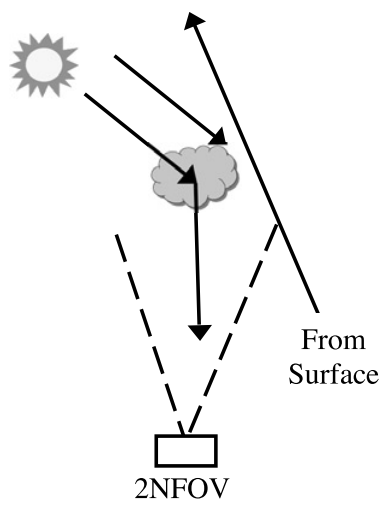

(a) (b)

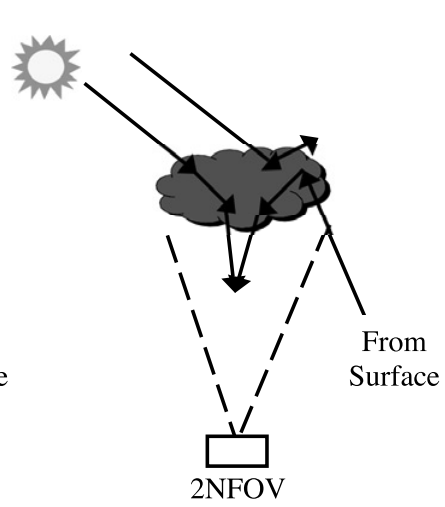

Figure 11. Schematic illustration of radiances received by the 2 NFOV radiometer for two cases: (a) the FOV is partially covered by thin clouds and (b) the FOV is fully covered by thick clouds. In both cases, the $2 \mathrm{NFOV}$ radiometer receives the same amount of radiation. 

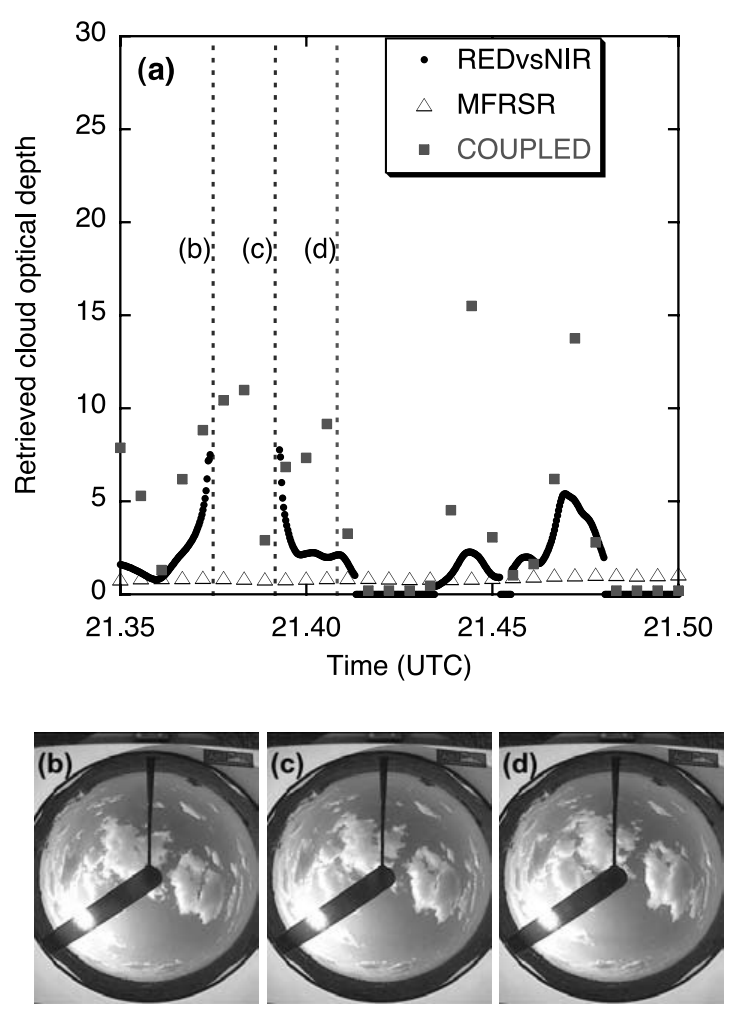

Figure 12. Same as Figure 7 but for 2121:002130:00 UTC, 29 September 2004. TSI snapshots were taken at (b) 2122:30, (c) 2123:30, and (d) 2124:30 UTC. Surface albedo in this case is dependent on solar zenith angle. Here $\rho_{\text {red }}=0.13, \rho_{\mathrm{NIR}}=0.28$, and SZA $=58^{\circ} \pm 1$.

these cumulus clouds. In contrast with REDvsNIR and MFRSR methods, the COUPLED method that combines radiance and flux works well for this type of clouds. It confirms the conclusion we draw in section 4.2 for the COUPLED method: the more broken clouds are, the more stable are the retrieved cloud optical depths. Indeed this method works best when the effective cloud fraction is much less 1 .

\section{Summary and Discussion}

[59] We conducted the first extensive field observations of two new methods to retrieve cloud optical properties in any cloud situation from patchy to overcast. These two retrieval methods are based on the fact that, while clouds have nearly identical optical properties at 673 (red) and $870 \mathrm{~nm}$ (NIR), vegetated surface reflectances at these two wavelengths are quite different (much brighter at the NIR wavelength). The observations were taken at the ARM Oklahoma site, from September to November 2004, using a new two-channel narrow-field-of-view (2NFOV) radiometer. Although the surrounding area was not perfectly green and vegetated during that time period, from this study we were able to learn the strengths and limitations of these two methods.

[60] The first method, called REDvsNIR, retrieves not only cloud optical depth but also effective cloud fraction. Because of 2NFOV 1-s resolution, the REDvsNIR method captured changes in cloud optical properties at the natural timescale of cloud evolution. Compared to the microwave cloud retrieval method, we found that for both overcast and broken clouds the average cloud optical depths of these two methods agreed to better than $15 \%$ with each other. Compared to the flux method, we found that because of cloud inhomogeneity, even for overcast case, the difference between the REDvsNIR and the flux methods could be as large as $50 \%$. The flux method is not supposed to work for broken clouds because the required plane-parallel assumption is violated.

[61] The REDvsNIR retrieval method is not perfect either. First of all, this method is also based on 1-D lookup tables rather than 3-D radiative transfer calculations. Its accuracy is affected by strong reflectance from cloud edges and it does not retrieve cloud fraction for small cloud optical depths. Secondly, we found that it retrieves incorrect cloud optical depths when the measurement FOV is not entirely filled with clouds. As a result, the FOV of the $2 \mathrm{NFOV}$ radiometer has been recently decreased to $1.2^{\circ}$. We expect that this reduction in the field of view should achieve less interference from clear-sky and cloud edge in our retrievals.

[62] The second retrieval method, called COUPLED, combines both radiance and flux measurements. Retrievals from this method in general agree to $15 \%$ with those from REDvsNIR. However, the COUPLED method is more sensitive to instrumental uncertainties and surface albedo than the REDvsNIR. When clouds are overcast and thick, and the spectral contrast in surface reflectance is small, the COUPLED method can become unstable and often substantially overestimates cloud optical depths. Its retrievals are more reliable for thinner and broken clouds.

[63] The ARM program deployed this newest 2NFOV radiometer in a field campaign at Point Reyes, California during June-September 2005. This site is ideal for the REDvsNIR and COUPLED methods since there is a much larger spectral contrast in surface reflectance than at the ARM Oklahoma site (see Figure 4). Since a substantial amount of data has been collected, for the future work, we plan to systematically evaluate cloud optical properties retrieved by these two methods in much more preferable conditions, and compare them with those from the flux and microwave methods.

[64] Acknowledgment. This research was supported by the Office of Science (BER, U.S. Department of Energy, interagency agreement DEAI02-95ER61961 and DE-FG02-05ER63955) as part of the ARM program.

\section{References}

Barker, H. W., and A. Marshak (2001), Inferring optical depth of broken clouds above green vegetation using surface solar radiometric measurements, J. Atmos. Sci., 58, 2989-3006.

Barker, H. W., T. J. Curtis, E. Leontieva, and K. Stamnes (1998), Optical depth of overcast cloud across Canada: Estimates based on surface pyranometer and satellite measurements, J. Clim., 11, 2980-2994.

Barker, H. W., C. F. Pavloski, M. Ovtchinnikov, and E. E. Clothiaux (2004), Assessing a cloud optical depth retrieval algorithm with modelgenerated data and the frozen turbulence assumption, J. Atmos. Sci., 61, $2951-2956$.

Beaulne, A., H. W. Barker, and J.-P. Blanchet (2005), Estimating cloud optical depth from surface radiometric observations: Sensitivity to instrument noise and aerosol contamination, J. Atmos. Sci., 62, 40954104.

Boers, R. (1997), Simultaneous retrievals of cloud optical depth and droplet concentration from solar irradiance and microwave liquid, J. Geophys. Res., 102, 2981-2989. 
Box, M. A., S. A. W. Gerstl, and C. Simmer (1988), Application of the adjoint formulation to the calculation of atmospheric radiative effects, Contrib. Atmos. Phys., 61, 303-311.

Dong, X., T. P. Ackerman, E. E. Clothiaux, P. Pilewskie, and Y. Han (1997), Microphysical and radiative properties of boundary layer stratiform clouds deduced from ground-based measurements, J. Geophys. Res., $102,23,829-23,843$.

Holben, B. N., et al. (1998), AERONET - A federated instrument network and data archive for aerosol characterization, Remote Sens. Environ., 66 , $1-16$.

Knyazikhin, Y., A. Marshak, and R. B. Myneni (2005), 3D radiative transfer in vegetation canopies and cloud-vegetation interaction, in Three-Dimensional Radiative Transfer in Cloudy Atmospheres, edited by A. Marshak and A. B. Davis, pp. 623-658, Springer, New York.

Leontieva, E., and K. Stamnes (1994), Estimations of cloud optical thickness from ground-based measurements of incoming solar radiation in the Arctic, J. Clim., 7, 566-578.

Leontieva, E., and K. Stamnes (1996), Remote sensing of cloud optica properties from ground-based measurements of transmittance: A feasibility case, J. Appl. Meteorol., 35, 2012-2022.

Liljegren, J. C., and B. M. Lesht (1996), Measurements of integrated water vapor and cloud liquid water from microwave radiometers at the DOE ARM Cloud and Radiation Testbed in the Southern Great Plains, paper presented at International Geoscience and Remote Sensing Symposium, Inst. of Electr. and Electron. Eng., Lincoln, Nebr., 21 - 26 May.

Liljegren, J. C., E. E. Clothiaux, G. G. Mace, S. Kato, and X. Dong (2001), A new retrieval for cloud liquid water path using a ground-based microwave radiometer and measurements of cloud temperature, J. Geophys. Res., 106, 14,485-14,500.

Luo, Y., A. P. Trishchenko, R. Latifovic, and Z. Li (2005), Surface bidirectional reflectance and albedo properties derived using a land cover-based approach with Moderate Resolution Imaging Spectroradiometer observations, J. Geophys. Res., 110, D01106, doi:10.1029/2004JD004741.

Marshak, A., Y. Knyazikhin, A. B. Davis, W. J. Wiscombe, and P. Pilewskie (2000), Cloud-vegetation interaction: Use of normalized difference cloud index for estimation of cloud optical thickness, Geophys. Res. Lett., 27, 1695-1698.

Marshak, A., Y. Knyazikhin, K. D. Evans, and W. J. Wiscombe (2004), The "RED versus NIR" plane to retrieve broken-cloud optical depth from ground-based measurements, cloud-vegetation interaction: Use of normalized difference cloud index for estimation of cloud optical thickness, J. Atmos. Sci., 61, 1911-1925.

Min, Q. L., and L. C. Harrison (1996), Cloud properties derived from surface MFRSR measurements and comparison with GOES results at the ARM SGP site, Geophys. Res. Lett., 23, 1641-1644.

Min, Q., E. Joseph, and M. Duan (2004), Retrievals of thin cloud optical depth from a multifilter rotating shadowband radiometer, J. Geophys. Res., 109, D02201, doi:10.1029/2003JD003964.

Petty, G. W. (Ed.) (2004), A First Course in Atmospheric Radiation, 444 pp., Sundog, Madison, Wis.

Ricchiazzi, P., C. Gautier, and D. Lubin (1995), Cloud scattering optical depth and local surface albedo in the Antarctic: Simultaneous retrieval using ground-based radiometry, J. Geophys. Res., 100, 21,091-21,104.

Schaaf, C. B., et al. (2002), First operational BRDF, albedo nadir reflectance products from MODIS, Remote Sens. Environ., 83, 135-148.

Stamnes, K., S.-C. Tsay, W. J. Wiscombe, and K. Jayaweera (1988), Numerically stable algorithm for discrete-ordinate-method radiative transfer in multiple scattering and emitting layered media, Appl. Opt., 27, 2502-2512.

Tucker, C. J. (1979), Red and photographic infrared linear combination for monitoring vegetation, Remote Sens. Environ., 8, 127-150.

H. W. Barker, Cloud Physics Research Division, Meteorological Service of Canada, 4905 Dufferin Street, Downsview, ON, Canada M3H 5T4.

J. C. Barnard, Pacific Northwest National Laboratory, PO Box 999 K9-30, Richland, WA 99352, USA

J. C. Chiu, Joint Center for Earth Systems Technology, University of Maryland Baltimore County, 1000 Hilltop Circle, Baltimore, MD 21250, USA. (cchiu@climate.gsfc.nasa.gov)

Y. Knyazikhin, Department of Geography, Boston University, 675 Commonwealth Ave., Boston, MA 02215, USA.

Y. Luo, Canada Centre for Remote Sensing, Natural Resources Canada, 588 Booth Street, Ottawa, ON Canada K1A 0 Y7.

A. Marshak and W. J. Wiscombe, Climate and Radiation Branch, NASA Goddard Space Flight Center, Code 613.2, Greenbelt, MD 20771, USA. 